\title{
Two Cases of Acute Mastoiditis with Subperiosteal Abscess
}

\author{
Sung Ryeal Kim, Oak-Sung Choo and Hun Yi Park \\ Department of Otolaryngology, Ajou University School of Medicine, Suwon, Korea
}

\author{
Received July 6, 2013 \\ Revised August 5, 2013 \\ Accepted August 12, 2013
}

\section{Address for correspondence Hun Yi Park, MD \\ Department of Otolaryngology, Ajou University School of Medicine, 164 Worldcup-ro, Yeongtong-gu, Suwon 443-721, Korea \\ Tel +82-31-219-5136 \\ Fax +82-31-219-5264 \\ E-mail hunyi@ajou.ac.kr}

The incidence of mastoiditis in pediatric age has consistently increased over the last two decades due to increase of antibiotic-resistant bacteria. Compared to young patients, occurrence of acute otitis media complications such as acute mastoiditis and subperiosteal abscess is relatively low in adults. Various treatments for acute mastoiditis with subperiosteal abscess such as tympanostomy tube insertion, intravenous antibiotics, and postauricular incision and drainage have avoided the morbidity and necessity of mastoid surgery. Recently, many studies have indicated mastoidectomy only in cases of severe complications or failure of disease improvement after antibiotic treatment and myringotomy. In this report, we present two cases of successful treatment of subperiosteal abscess and discuss the management of acute mastoiditis with subperiosteal abscess in both child and adult.

Korean J Audiol 2013;17:97-100

KEY WORDS: Subperiosteal abscess · Acute mastoiditis · Mastoidectomy.

\section{Introduction}

Acute coalescent mastoiditis is known as a complication of acute otitis media. Development of antibiotics led to rapid decrement of acute mastoiditis, reporting its incidence as low as $0.004 \%$ in the $1980 \mathrm{~s} .{ }^{1)}$ However, acute otitis media associated with suppurative complications such as subperiosteal abscess, facial palsy, and intracranial complication are yet on a constant rise due to recent increase of antibiotic-resistant bacteria. ${ }^{2-6)}$ Several studies have reported satisfying treatment outcomes of acute coalescent mastoiditis with proper drainage, ventilation and intravenous antibiotics although other authors have questioned the necessity of mastoidectomy.

Here, we report our experience in treatment of acute otitis media-induced acute mastoiditis associated with subperiosteal abscess in child and adult, discuss proper management of the disease, and review its literature.

\section{Case Report}

\section{Case 1 (Child)}

A 2 year old female admitted to the emergency department due to 2 days of right postauricular area swelling. The patient was previously treated with antibiotics for a week at a local clinic for acute otitis media. Despite antibiotic treatment, the patient suffered from right otalgia and fever upon admission.
Through physical examination, bulging of the tympanic membrane and erythematous change and swelling of the right postauricular area with tenderness were noticed. Further evaluation by temporal bone CT revealed soft tissue density filling the right middle ear and mastoid cavity with erosion of the cortical bone along with formation of subperiosteal abscess (Fig. 1). Under the impression of acute mastoiditis with subperiosteal abscess, percutaneous aspiration was performed on the postauricular area resulting in $3 \mathrm{cc}$ of purulent fluid. The procedure was followed by incision and drainage (I \& D) and oral antibiotics were prescribed to the patient. On third day after I $\& \mathrm{D}$, the patient showed persistent redness, tenderness, and swelling of the postauricular area (Fig. 2) and injected bulging tympanic membrane, regardless of the treatment. Therefore, it was postulated that I \& D and oral antibiotics were inadequate and the patient was admitted to our department for intravenous antibiotics therapy.

On the second day of hospitalization, the patient underwent surgical treatment of right simple mastoidectomy and ventilation tube insertion because of consistent symptoms and physical findings in spite of 2 days of intravenous antibiotic treatment. Intraoperative finding showed massive granuloma beneath the postauricular area and in the mastoid cavity (Fig. 3). Erosion of cortical bone was also noticed after Palva flap elevation. Intraoperative culture study resulted in Streptococcus pneumoniae which was only susceptible to vancomycin. In- 
travenous antibiotic treatment with vancomycin was immediately initiated and the child was discharged after 13 days of surgery without any postoperative complications. During the 5 month follow up, the patient showed no signs or symptoms of recurrence.

\section{Case 2 (Adult)}

A 41 year old male visited the emergency department suffering from right otalgia for 1 month. The patient had no known medical history and complained of hearing impairment and otalgia on the right side. Physical examination showed injected bulging tympanic membrane associated with right postauricular swelling, redness and tenderness (Fig. 4). Further evaluation through pure tone audiometry revealed a mixed hearing loss on the right side with $60 \mathrm{~dB}$ air conduction level and 30 $\mathrm{dB}$ air-bone gap. Radiologic examinations of temporal bone CT and MRI showed soft tissue density filling the right middle ear and mastoid cavity with cortical bone erosion. Subperiosteal abscess was also detected over the cortical bone (Fig. 5). The patient was admitted and treated with I \& D and antibiotics. Culture study that was processed during I \& D revealed penicillin-sensitive Streptococcus pneumoniae. Even after 7 days of hospitalization, there were no signs of improvement. Thus, on the 8th day of hospitalization, right canal wall up mastoidectomy and tympanoplasty were performed. During the operation, granulation tissue in the mastoid cavity and middle

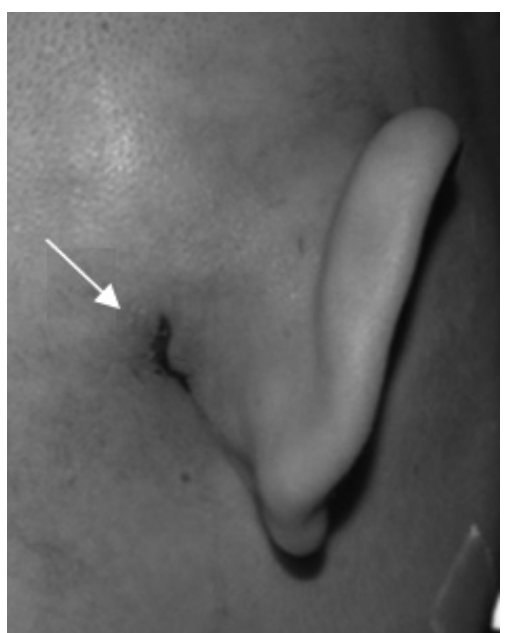

Fig. 2. Photograph of retro-auricular area after 3 days of incision \& drainage. Soft tissue swelling with erythematous change is noticed on the patient's right post auricular area. Right auricle is protruded outward and downward. Previous incision \& drainage site is also present (white arrow).
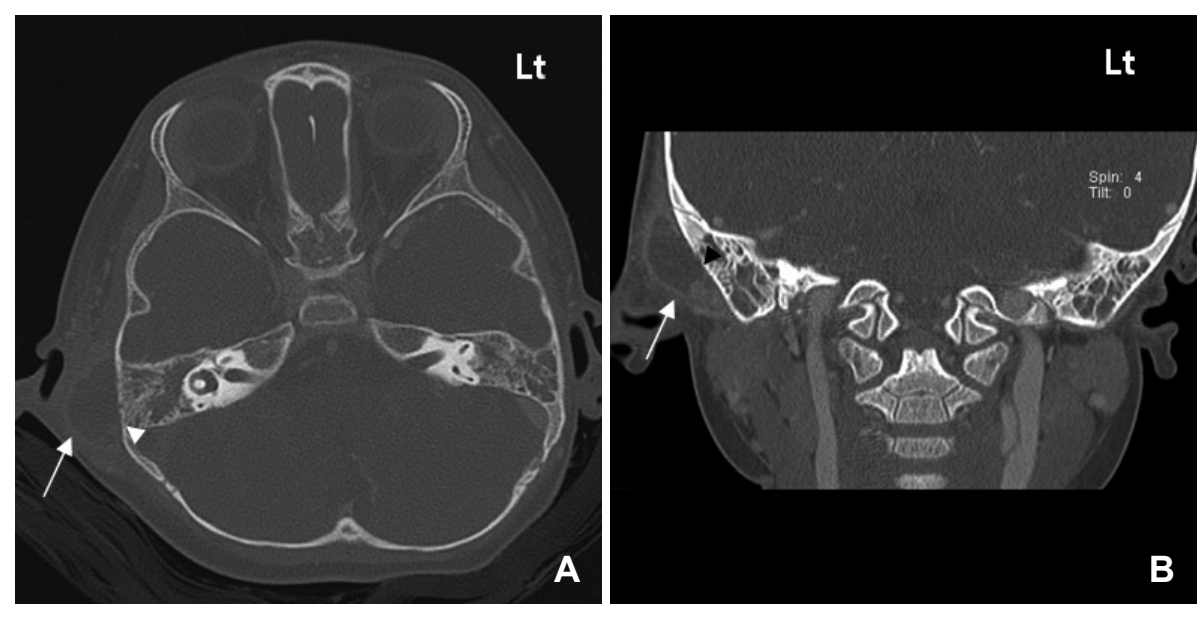

Fig. 1. Initial temporal bone computed tomography. Area of lucency representing abscess cavity is shown overlying right mastoid cortex (white arrows). Note the erosion of the mastoid cortex (white arrowhead). A: Axial view. B: Coronal view.
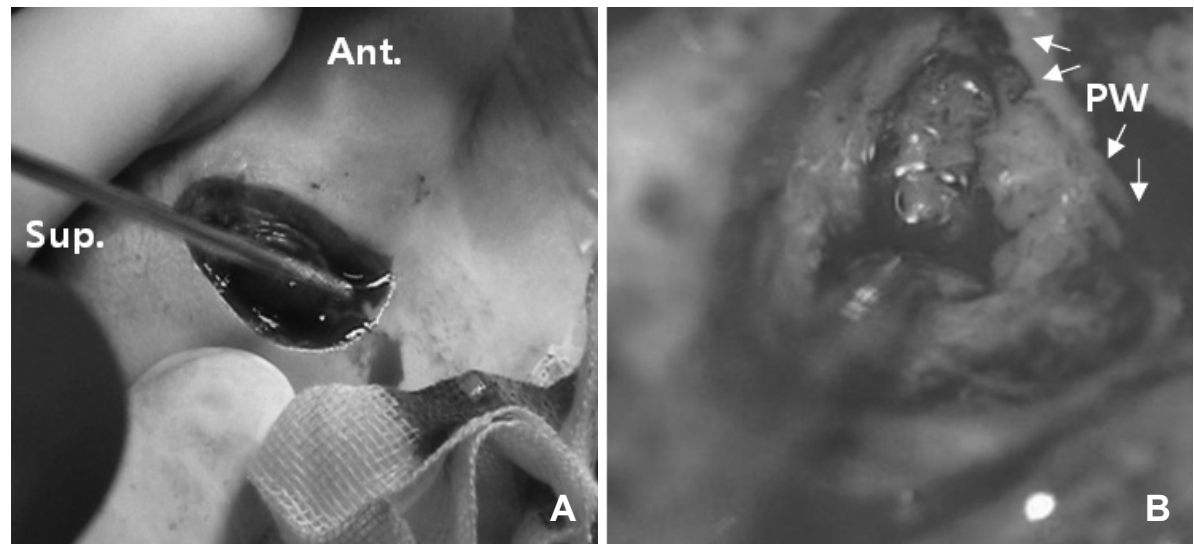

Fig. 3. A: Intraoperative findings. Retroauricular skin incision was made and profuse granulation tissue was present in subcutaneous layer. B: Mastoid antrum was filled with granulation tissue similar to findings of subcutaneous layer. PW: posterior external auditory canal wall. 
ear cavity was detected and removed completely (Fig. 6). The patient was discharged after a week of surgery with significant improvement of previous symptoms and physical findings. No suspicious signs of recurrence were encountered during the 1 year follow up.

\section{Discussion}

Acute mastoiditis with subperiosteal abscess frequently occurs as a complication of acute otitis media and the incidence rate had decreased in accordance with the development of antibiotics. However, in the past 20 years it is on the rise again probably due to the growth of antibiotics-resistant bacteria. ${ }^{2-6)}$

Generally, subperiosteal abscess occurs in children who had been diagnosed with acute otitis media. The disease may be suspected through its characteristic features of injected bulging tympanic membrane, persistent otalgia and fever despite oral antibiotics eventually advancing to postauricular swelling, erythematous change, and tenderness. Temporal bone CT uniquely features soft tissue density in the middle ear and mas-

Fig. 4. Clinical features of patient. A: A bulging and hyperemic tympanic membrane is noted. B: Soft tissue swelling, erythematous change and tenderness is present on patient's right postauricular area.
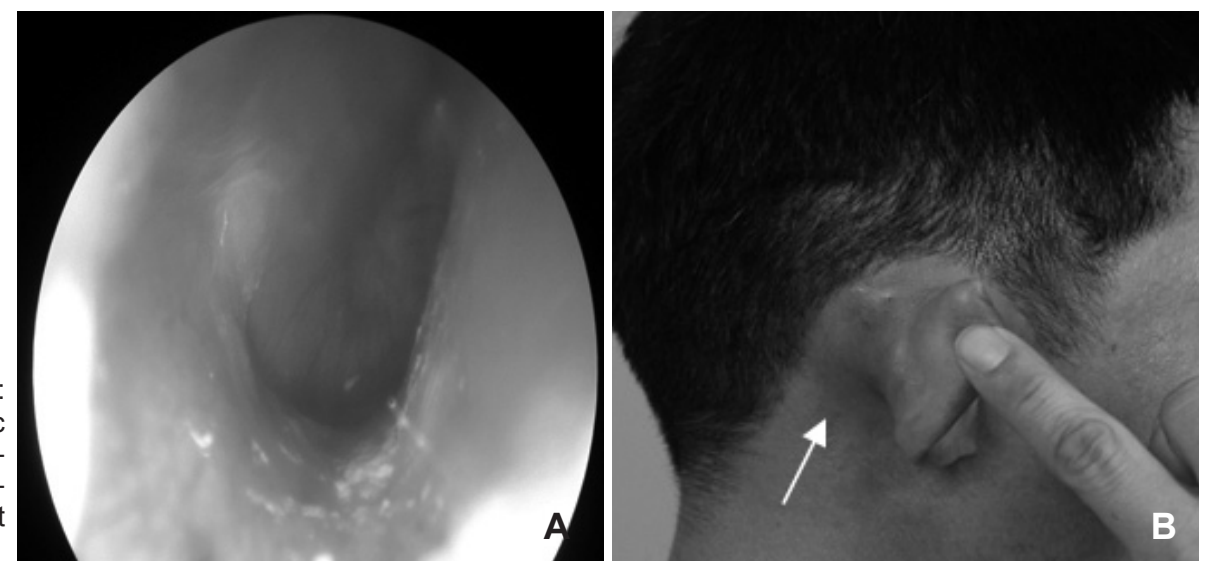

Fig. 5. Gadolinium-enhanced T1 weighted magnetic resonance images of the temporal bone. Inflammatory changes associated with pus like discharges in right middle ear, mastoid antrum and postauricular area are detected. Abscess with combined hemorrhage of right postauricular area is suspected through T1WI. A: Axial view. B: Coronal view. T1WI: T1-weighted image.
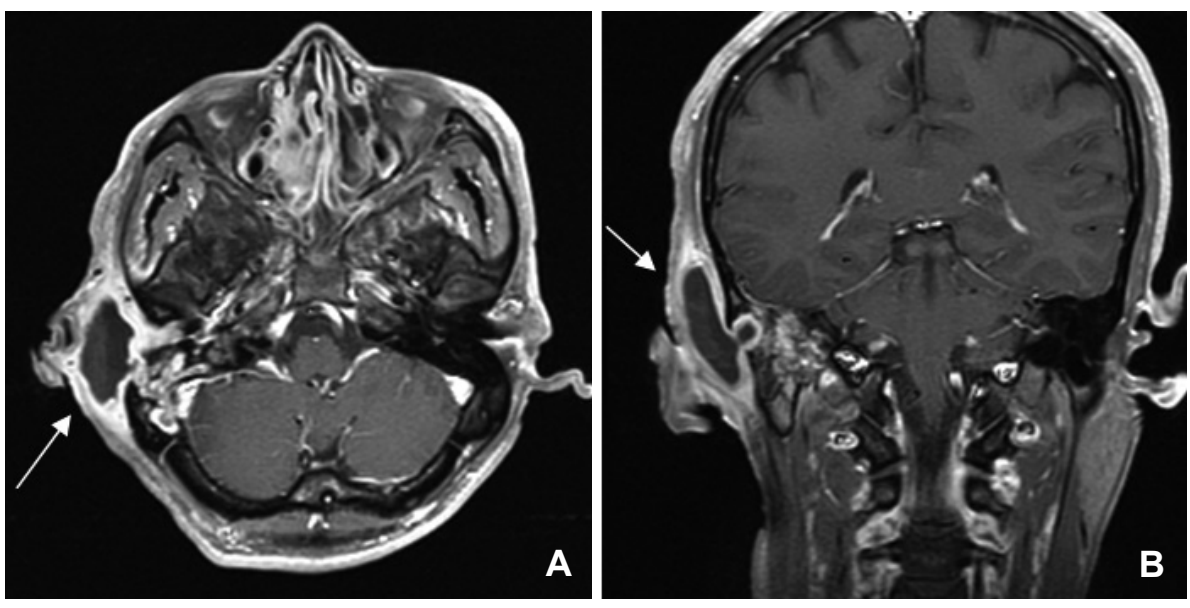

Fig. 6. Intraoperative findings. A: The retroauricualr skin incision was made with abundant granulation tissue noted in subcutaneous layer. B: After Palva flap elevation, cortical bone defect was recognized. TM: temporalis muscle, PW: posterior external auditory canal wall.
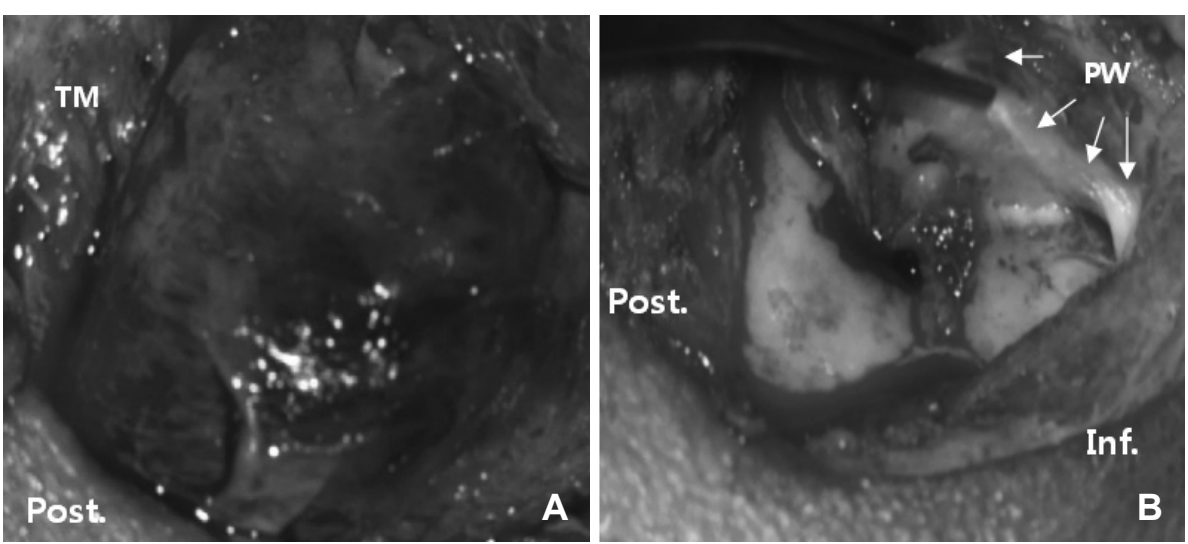
toid cavity, erosion or defect of cortical bone of the mastoid cavity, and contrast-enhanced subperiosteal shadows. In some cases, MRI may be necessary to determine intracranial complications. ${ }^{5}$ Similarly, in the second case, MRI was performed to detect possible intracranial involvement and differentiate other diagnosis since the occurrence rate of acute otitis media associated with complications is relatively low in adults.

Before the development of antibiotics, surgery was the treatment of choice for acute mastoiditis. Early surgical management and the use of proper antibiotics confirmed by culture study had been advocated to reduce morbidity and mortality especially in cases of acute mastoiditis associated with complications.

Recently, many studies have proved that proper I \& D, ventilation, and intravenous antibiotic are sufficient treatment methods for acute mastoiditis. Favorable outcomes have been reported after treatment of patients under 2 years of age through ventilation tube insertion, I \& D, and aspiration combined with intravenous antibiotics. ${ }^{7-10)}$ These less invasive treatment modalities should be considered before mastoidectomy to reduce anesthetic risks and due to concerns of possible adverse effects of the ventilation system in the temporal bone by early mastoidectomy. However, the surgical treatment should be considered in cases of disappointing treatment outcomes with intravenous antibiotics, I \& D, myringotomy or ventilation tube insertion. Mostly, studies have reported to proceed operation after 48 to 72 hours of conservative treatment. ${ }^{6-10)}$ The most common causative organism of acute mastoiditis is Streptococcus pneumoniae ${ }^{2,4,9)}$ which is often reported to be resistant to certain antibiotics. Therefore, proper antibiotic selection is also of great importance. In the first case, simple mastoidectomy was performed after 3 days from the patient's emergency room visit due to her exacerbating condition even after I \& D and oral antibiotics. As in this case, surgical intervention might be considered when there is no clinical improvement within 2-3 days after I \& D and antibiotic treatment. Other indications for simple mastoidectomy and ventilation tube insertion suggested in several studies ${ }^{11)}$ are 1) patient over 30 months old and $15 \mathrm{~kg}$, present with protruded mastoid abscess 2) associated with intracranial co-morbidities 3) diagnosed with cholesteatoma, and 4) failure of 2 week antibiotic treatment in patients with granuloma and suppurative otorrhea.

In the second case, the adult patient also underwent mas- toidectomy because the patient's condition did not adequately respond to I \& D and intravenous antibiotics. Acute coalescent mastoiditis in adults is often associated with chronic otitis media or cholesteatoma ${ }^{12)}$ that mastoidectomy is also known to be performed to determine other possible causes of acute mastoiditis.

In conclusion, the clinician should always be aware that patients diagnosed with acute otitis media may be accompanied by suppurative complication regardless of age. In addition, early diagnosis and treatment should be carried out with assistance of its characteristic clinical manifestations and unique image findings. Treatment modalities include administration of intravenous antibiotics, pain control, and early consideration of myringotomy, ventilation tube insertion, or I \& D. If the treatment effect is in question, mastoidecomy should be performed to prevent possible progression and avoid additional complications.

\section{REFERENCES}

1) Palva T, Virtanen H, Mäkinen J. Acute and latent mastoiditis in children. J Laryngol Otol 1985;99:127-36.

2) Benito MB, Gorricho BP. Acute mastoiditis: increase in the incidence and complications. Int J Pediatr Otorhinolaryngol 2007;71:1007-11.

3) Gliklich RE, Eavey RD, Iannuzzi RA, Camacho AE. A contemporary analysis of acute mastoiditis. Arch Otolaryngol Head Neck Surg 1996;122:135-9.

4) Thorne MC, Chewaproug L, Elden LM. Suppurative complications of acute otitis media: changes in frequency over time. Arch Otolaryngol Head Neck Surg 2009;135:638-41.

5) Vera-Cruz P, Farinha RR, Calado V. Acute mastoiditis in children-our experience. Int J Pediatr Otorhinolaryngol 1999;50:113-7.

6) Migirov L, Yakirevitch A, Kronenberg J. Mastoid subperiosteal abscess: a review of 51 cases. Int J Pediatr Otorhinolaryngol 2005;69: 1529-33.

7) Harley EH, Sdralis T, Berkowitz RG. Acute mastoiditis in children: a 12-year retrospective study. Otolaryngol Head Neck Surg 1997;116: 26-30.

8) Luntz M, Brodsky A, Nusem S, Kronenberg J, Keren G, Migirov L, et al. Acute mastoiditis--the antibiotic era: a multicenter study. Int J Pediatr Otorhinolaryngol 2001;57:1-9.

9) Bauer PW, Brown KR, Jones DT. Mastoid subperiosteal abscess management in children. Int J Pediatr Otorhinolaryngol 2002;63:185-8.

10) Lahav J, Handzel O, Gertler R, Yehuda M, Halperin D. Postauricular needle aspiration of subperiosteal abscess in acute mastoiditis. Ann Otol Rhinol Laryngol 2005;114:323-7.

11) Zanetti D, Nassif N. Indications for surgery in acute mastoiditis and their complications in children. Int J Pediatr Otorhinolaryngol 2006; 70:1175-82.

12) Leskinen K, Jero J. Acute complications of otitis media in adults. Clin Otolaryngol 2005;30:511-6. 